\title{
Recomendaciones nutricionales para el consumo de carne roja y procesada teniendo en cuenta las preferencias poblacionales
}

Nutritional recommendations for the consumption of red and processed meat taking into account population's preferences

\section{Comentado de:}

Johnston B y col. Ann Intern Med. 2019;171(10):756-764. doi: 10.7326/M19-1621'.

\section{Introducción}

Existen guías en la literatura contemporánea que recomiendan limitar el consumo de carne roja (procesada y no procesada) ${ }^{2-6}$. Incluso, la Sociedad Americana contra el Cáncer y la Agencia Internacional de Investigación sobre el Cáncer de la Organización Mundial de la Salud han indicado que el consumo de carne roja es "probablemente carcinogénico"para los humanos, mientras que la carne procesada es considerada un "carcinógeno". Sin embargo, estas recomendaciones están basadas principalmente en estudios observacionales, limitados en establecer inferencia causal.

\section{Objetivo}

Emitir recomendaciones nutricionales sobre la base de la mejor evidencia disponible respecto de la asociación entre el consumo de carne roja y/o procesada y la incidencia de eventos cardiovasculares y cáncer.

\section{Revisión de la evidencia y desarrollo de la guía}

Se formularon dos preguntas PICO: 1) En adultos, ¿cuál es el efecto de las dietas y hábitos alimentarios bajos en carnes rojas o procesadas comparados con dietas y hábitos alimentarios con alto contenido de carnes rojas o procesadas en el riesgo de desarrollar desenlaces importantes en los miembros de la comunidad?; y 2) ¿Cuáles son los valores y preferencias relacionados con la salud respecto del consumo de carnes rojas y procesadas?

Para responder a la primera, se realizaron cuatro revisiones sistemáticas que incluyeron ensayos clínicos aleatorizados y estudios de cohorte, con al menos 1.000 participantes (de 18 años o más), seguidos por un período mínimo de seis meses. Estos estudios evaluaron dietas con cantidades variables de carne roja y carne procesada. Se excluyeron aquellos cuya muestra estuviera formada por $20 \%$ o más de personas embarazadas, participantes con cáncer o alguna condición crónica preexistente (diferente a la enfermedad cardiometabólica).

Para responder la segunda pregunta se realizó una revisión sistemática a partir de estudios cualitativos (entrevistas y grupos focales) y cuantitativos (estudios de corte transversal).

La búsqueda bibliográfica se realizó en las bases de datos más importantes (no se explicitan en el texto del artículo original), desde sus orígenes hasta julio 2018, sin restricción de lenguaje. Para el caso de MEDLINE, la búsqueda se extendió hasta abril de 2019.
Se utilizó el marco GRADE para pasar de la evidencia a las recomendaciones. El panel de expertos incluyó 11 profesionales de la salud y tres miembros de la comunidad, todos residentes de países de altos ingresos (Canadá, Inglaterra, Alemania, Nueva Zelanda, Polonia, España y Estados Unidos).

\section{Intervenciones y/o exposiciones de interés}

Reducción de tres porciones de carne roja o procesada por semana respecto al consumo habitual basal de la población de Norteamérica y Europa Occidental, o adherencia a un patrón o hábito alimentario bajo en consumo de carnes rojas o procesadas.

\section{Medición de resultados principales}

Se evaluaron la mortalidad por todas las causas, los eventos cardiometabólicos mayores (infarto de miocardio, accidente cerebrovascular y diabetes) y la incidencia y mortalidad de cáncer de próstata, ginecológico y gastrointestinal.

\section{Resultados}

En la Tabla 1 se muestran los resultados principales relacionados con los eventos cardiometabólicos y el cáncer.

Respecto de los valores y preferencias en relación al consumo de carnes, las personas omnívoras suelen mantenerse apegadas al consumo de carne; informaron que disfrutan de comer carne que la consideran un componente esencial de una dieta saludable, y que en ocasiones se sienten limitados para cocinar platos vegetarianos. Muchos expresan además poca voluntad para disminuir el consumo de carnes, y desconfianza respecto de la información científica acerca de sus riesgos potenciales. Entre los vegetarianos encuestados o los omnívoros que ya habían reducido el consumo de carnes, aparecen frecuentemente razones relacionadas con la salud.

\section{Conclusión / recomendaciones}

No hay evidencia suficiente para demostrar la causalidad de la asociación entre el cáncer y los eventos cardiometabólicos mayores con el consumo de carne roja o procesada. Por lo tanto, el panel recomienda que los adultos continúen con el consumo habitual de carne, según sus valores y preferencias individuales (recomendación débil, bajo grado de evidencia).

Fuente de financiamiento/Conflictos de interés de los autores: $E$ desarrollo de la recomendación no recibió ninguna fuente de financiamiento externo. Uno de los autores del artículo recibió financiamiento en forma de becas de entidades públicas (São Paulo Research Foundation, National Council for Scientific and Technological Development, Facultad de Medicina, Universidad Dalhousie). 
Tabla 1. Evaluación de la inferencia causal basada en los efectos estadísticamente significativos asociados a la reducción (tres porciones semanales menos) en el consumo de carne roja o de carne procesada o la adherencia a un patron alimentario bajo en consumo de carnes. Notas: *Seguimiento hipotético por 10,8 años. ${ }^{*}$ Seguimiento hipotético de por vida.

\begin{tabular}{|c|c|c|c|c|c|c|}
\hline \multirow[t]{2}{*}{ Evento } & \multicolumn{2}{|c|}{ Carne roja } & \multicolumn{2}{|c|}{ Carne procesada } & \multicolumn{2}{|c|}{ Patrón alimentario } \\
\hline & $\begin{array}{l}\text { Diferencia de } \\
\text { riesgo (rango) }\end{array}$ & $\begin{array}{l}\text { Grado de } \\
\text { evidencia }\end{array}$ & $\begin{array}{l}\text { Diferencia de } \\
\text { riesgo (rango) }\end{array}$ & $\begin{array}{l}\text { Grado de } \\
\text { evidencia }\end{array}$ & $\begin{array}{l}\text { Diferencia de } \\
\text { riesgo (rango) }\end{array}$ & $\begin{array}{l}\text { Grado de } \\
\text { evidencia }\end{array}$ \\
\hline $\begin{array}{l}\text { Mortalidad } \\
\text { cardiovascu- } \\
\text { lar }^{\star}\end{array}$ & $\begin{array}{c}4 \text { menos/ } \\
1.000 \\
\text { personas (5 a } \\
4 \text { menos) }\end{array}$ & $\begin{array}{r}\text { Muy baja } \\
\oplus \ominus \ominus \ominus\end{array}$ & $\begin{array}{c}1 \text { a } 12 \text { menos/ } \\
1.000 \\
\text { personas ( } 7 \text { a } \\
1 \text { menos) }\end{array}$ & $\begin{array}{r}\text { Muy baja } \\
\oplus \ominus \ominus \ominus\end{array}$ & $\begin{array}{c}6 \text { menos } / 1.000 \\
\text { personas }(9 \text { a } \\
2 \text { menos })\end{array}$ & $\begin{array}{l}\text { Muy baja } \\
\qquad \oplus \ominus \ominus \ominus\end{array}$ \\
\hline $\begin{array}{l}\text { Diabetes tipo } \\
2^{\star}\end{array}$ & $\begin{array}{c}6 \text { menos/ } \\
1.000 \\
\text { personas }(7 \mathrm{a} \\
4 \text { menos) } \\
\end{array}$ & $\begin{array}{l}\text { Baja } \\
\qquad \oplus \ominus \ominus\end{array}$ & $\begin{array}{c}12 \text { menos/ } \\
1.000 \\
\text { personas (16 a } \\
9 \text { menos) } \\
\end{array}$ & $\begin{array}{r}\text { Muy baja } \\
\oplus \ominus \ominus \ominus\end{array}$ & $\begin{array}{c}14 \\
\text { menos/1.000 } \\
\text { personas (18 a } \\
8 \text { menos) } \\
\end{array}$ & $\begin{array}{l}\text { Muy baja } \\
\qquad \oplus \ominus \ominus \ominus\end{array}$ \\
\hline $\begin{array}{l}\text { Mortalidad } \\
\text { por cáncer }^{\star \star}\end{array}$ & $\begin{array}{c}7 \text { menos/ } \\
1.000 \\
\text { personas (9 a } \\
6 \text { menos) }\end{array}$ & $\begin{array}{l}\text { Baja } \\
\oplus \oplus \ominus \ominus\end{array}$ & $\begin{array}{c}8 \text { menos/ } \\
1.000 \\
\text { personas }(12 \mathrm{a} \\
6 \text { menos })\end{array}$ & $\begin{array}{l}\text { Baja } \\
\oplus \oplus \ominus \ominus\end{array}$ & $\begin{array}{c}12 \\
\text { menos } / 1.000 \\
\text { personas (18 a } \\
4 \text { menos) }\end{array}$ & $\begin{array}{l}\text { Muy baja } \\
\qquad \oplus \ominus \ominus \ominus\end{array}$ \\
\hline
\end{tabular}

\section{Comentario}

En la actualidad, cada vez más personas son conscientes del modo en el que su alimentación afecta su vida y optan, por razones éticas o médicas, por dietas libres de contenido de origen animal. Ya sea por la importancia cultural que tiene el consumo de carne en Argentina, o porque es una consulta que aparece cada vez con mayor frecuencia en el consultorio, el análisis crítico de esta guía podría brindar herramientas a la hora de manejar este motivo de consulta en el ámbito ambulatorio de la atención primaria de la salud. En Argentina, según un estudio realizado por la Sociedad Rural en $2005^{7}$, el $97 \%$ de la población mayor de 14 años consume carne vacuna, y el segundo dato revelador es que nueve de cada diez hogares e individuos son consumidores "habituales", entendiendo por habitual una ingesta de carne vacuna dos días a la semana o más. En cuanto a los valores y preferencias de la población, hay una tendencia mayoritaria entre los consumidores a considerar a la carne como el centro indispensable de la comida, reconociendo a los otros grupos de alimentos como un rol de acompañamiento y/o complementario en el aporte de nutrientes.

Las guías de recomendaciones nutricionales son desafiantes porque cada fuente potencial de evidencia tiene limitaciones sustanciales. Los ensayos aleatorizados están limitados por el tamaño de la muestra, la duración del seguimiento y las dificultades que los participantes tienen para cumplir con las dietas prescriptas. Estas limitaciones hacen que mostrar el efecto de una intervención sea muy difícil. Por otro lado, la evidencia proveniente de estudios observacionales está limitada por la inevitable confusión residual, una vez ajustados sus resultados.

En esta guía de práctica clínica, los ensayos aleatorizados presentan una diferencia muy pequeña en el consumo de carne en comparación con el grupo control, mientras que los estudios observacionales se ven limitados principalmente por la falta de precisión en los registros dietarios (sesgo de información). Por estos motivos, se jerarquiza que la toma de decisiones sobre el consumo de carne dependa principalmente de los valores y las preferencias individuales.

Por otra parte, el panel se centró en los desenlaces en la salud que se cree están asociados con el consumo de carne roja y carne procesada. No se tuvieron en cuenta el maltrato animal ni la contaminación ambiental relacionados con el consumo de carne al hacer las recomendaciones. Por lo tanto, estas revisiones pueden tener una relevancia limitada para las personas que atribuyen gran importancia a estos aspectos.

\section{Conclusiones de la comentadora}

Es bien conocida la dificultad metodológica para generar evidencia de buena calidad sobre epidemiología nutricional. En esta temática, en la que abundan las recomendaciones no basadas en evidencia, es valorable la confección de revisiones sistemáticas, a pesar del alto riesgo de confundidores y sesgos imposibles de corregir. La emisión de una recomendación débil abre la puerta a la toma de decisiones compartidas sobre continuar o no continuar consumiendo carne roja, en un medio donde empieza a difundirse cada vez más la alimentación basada en plantas. 
Antonella Stellardo [ Servicio de Medicina Familiar y Comunitaria, Hospital Italiano de Buenos Aires. antonella.stellardo@hospitalitaliano.org.ar ]

Stellardo A. Recomendaciones nutricionales para el consumo de carne roja y procesada teniendo en cuenta las preferencias poblacionales . Evid Actual Pract Ambul. 2020;23(4):e002080. Comentado de: Johnston B y col. Unprocessed Red Meat and Processed Meat Consumption: Dietary Guideline Recommendations From the Nutritional Recommendations (NutriRECS) Consortium. Ann Intern Med. 2019;171(10):756-764. doi: 10.7326/M19-1621. PMID: 31569235

\section{Referencias}

1. Johnston BC, Zeraatkar D, Han MA, et al. Unprocessed Red Meat and Processed Meat Consumption: Dietary Guideline Recommendations From the Nutritional Recommendations (NutriRECS) Consortium. Ann Intern Med. 2019;171(10):756-764. Available from: 10.7326/M19-1621.

2. U S Department of Health and Human and Services U S Department of Agriculture. 2015-2020 Dietary Guidelines for Americans; 2015. Available from: https://health.gov/sites/default/files/2019-09/2015-2020_Dietary_Guidelines.pdf.

3. United Kingdom. National Health Institutes. The Eatwell Guide; 2019. Available from: https://www.nhs.uk/live-well/eat-well/the-eatwell-guide/.

4. World Cancer Research Fund. Continuous Update Project Expert Report 2018. Meat, fish and dairy products and the risk of cancer; 2018. Available from: https://www.wcrf.org/sites/default/files/Meat-Fish-and-Dairy-products.pdf.

5. Rock CL, Thomson C, Gansler T, et al. American Cancer Society guideline for diet and physical activity for cancer prevention. CA Cancer J Clin . 2020;70(4):245-271. Available from: 10.3322/caac.21591.

6. Bouvard V, Loomis D, Guyton KZ, et al. Carcinogenicity of consumption of red and processed meat. Lancet Oncol. 2015;16(16):1599-1600. Available from: 10.1016/S1470-2045(15)00444-1.

7. Argentina TG. El consumo de carne vacuna en la argentina; 2005. 